\title{
Classes of measures closed under mixing and convolution. Weak stability
}

\author{
by \\ J. K. Misiewicz (Zielona Góra), K. Oleszkiewicz (Warszawa) \\ and K. URBANIK (Wrocław)
}

\begin{abstract}
For a random vector $X$ with a fixed distribution $\mu$ we construct a class of distributions $\mathcal{M}(\mu)=\{\mu \circ \lambda: \lambda \in \mathcal{P}\}$, which is the class of all distributions of random vectors $X \Theta$, where $\Theta$ is independent of $X$ and has distribution $\lambda$. The problem is to characterize the distributions $\mu$ for which $\mathcal{M}(\mu)$ is closed under convolution. This is equivalent to the characterization of the random vectors $X$ such that for all random variables $\Theta_{1}, \Theta_{2}$ independent of $X, X^{\prime}$ there exists a random variable $\Theta$ independent of $X$ such that

$$
X \Theta_{1}+X^{\prime} \Theta_{2} \stackrel{d}{=} X \Theta .
$$

We show that for every $X$ this property is equivalent to the following condition:

$$
\forall a, b \in \mathbb{R} \exists \Theta \text { independent of } X, \quad a X+b X^{\prime} \stackrel{d}{=} X \Theta .
$$

This condition reminds the characterizing condition for symmetric stable random vectors, except that $\Theta$ is here a random variable, instead of a constant.

The above problem has a direct connection with the concept of generalized convolutions and with the characterization of the extreme points for the set of pseudo-isotropic distributions.
\end{abstract}

1. Introduction. Let $\mathbb{E}$ be a separable real Banach space. By $\mathcal{P}(\mathbb{E})$ we denote the set of all Borel probability measures on $\mathbb{E}$. For $\mathbb{E}=\mathbb{R}$ we will use the simplified notation $\mathcal{P}(\mathbb{R})=\mathcal{P}$, and the set of all probability measures on $[0, \infty)$ will be denoted by $\mathcal{P}_{+}$. For every $a \in \mathbb{R}$ and every probability measure $\mu$, we define the rescaling operator $\mu \mapsto T_{a} \mu$ by the formula $\left(T_{a} \mu\right)(A)=\mu(A / a)$ when $a \neq 0$, and $T_{0}(\mu)=\delta_{0}$. This means that $T_{a} \mu$ is the distribution of the random vector $a X$ if $\mu$ is the distribution of the vector $X$. For every $\mu \in \mathcal{P}(\mathbb{E})$ and $\lambda \in \mathcal{P}$ we define a scale mixture $\mu \circ \lambda$

2000 Mathematics Subject Classification: Primary 60E05.

Key words and phrases: convolution, generalized convolution, pseudo-isotropic distributions, elliptically contoured distributions, weakly stable measures.

The research of the second named author was partially supported by the Polish KBN Grant 2 P03A 02722. 
of the measure $\mu$ with respect to the measure $\lambda$ by the formula

$$
(\mu \circ \lambda)(A)=\int_{\mathbb{R}}\left(T_{a} \mu\right)(A) \lambda(d a) .
$$

It is easy to see that $\mu \circ \lambda$ is the distribution of the random vector $X \Theta$ if $X$ and $\Theta$ are independent, $X$ has distribution $\mu$, and $\Theta$ has distribution $\lambda$.

We consider the set of all mixtures of the measure $\mu$, i.e.

$$
\mathcal{M}(\mu)=\{\mu \circ \lambda: \lambda \in \mathcal{P}\}=\mathcal{P} \circ \mu .
$$

When it is more convenient we will write $\mathcal{M}(\widehat{\mu})$ instead of $\mathcal{M}(\mu)$. The corresponding set of characteristic functions is denoted by

$$
\Phi(\mu)=\{\widehat{\nu}: \nu=\mu \circ \lambda, \lambda \in \mathcal{P}\}=\left\{\varphi: \varphi(\xi)=\int \widehat{\mu}(\xi t) \lambda(d t), \lambda \in \mathcal{P}, \xi \in \mathbb{E}^{*}\right\} .
$$

The problem discussed here has a very elementary formulation: characterize those probability measures $\mu$ on $\mathbb{E}$ for which the set $\mathcal{M}(\mu)$ is closed under convolution, i.e.

$$
\forall \nu_{1}, \nu_{2} \in \mathcal{M}(\mu), \quad \nu_{1} * \nu_{2} \in \mathcal{M}(\mu) .
$$

In the language of random vectors, this condition looks even simpler: Let $X, X^{\prime}, \Theta_{1}, \Theta_{2}$ be independent, where $X$ and $X^{\prime}$ have distribution $\mu$. If condition (A) holds, then there exists a random variable $\Theta$ independent of $X$ such that

$$
X \Theta_{1}+X^{\prime} \Theta_{2} \stackrel{d}{=} X \Theta .
$$

In particular, under the previous assumptions,

$$
\forall a, b \in \mathbb{R} \exists \Theta=\Theta(a, b), \quad X \text { and } \Theta \text { independent and } a X+b X^{\prime} \stackrel{d}{=} X \Theta .
$$

The main result of this paper states that condition (A) is equivalent to

$$
\forall a, b \in \mathbb{R}, \quad T_{a} \mu * T_{b} \mu \in \mathcal{M}(\mu) .
$$

EXAmple 1. The class of symmetric distributions on $\mathbb{R}$ is closed under mixing and under convolution. It is easy to see that this class can be written as $\mathcal{M}(\tau)$ for $\tau=\frac{1}{2} \delta_{1}+\frac{1}{2} \delta_{-1}$. Checking (B) in this case is especially simple. In the language of characteristic functions we have

$$
\begin{aligned}
\widehat{\tau}(a t) \widehat{\tau}(b t) & =\cos (a t) \cos (b t)=\frac{1}{2} \cos ((a+b) t)+\frac{1}{2} \cos ((a-b) t) \\
& =\int_{\mathbb{R}} \cos (t s)\left(\frac{1}{2} \delta_{a+b}+\frac{1}{2} \delta_{a-b}\right)(d s),
\end{aligned}
$$

which means that we can take $\frac{1}{2} \delta_{a+b}+\frac{1}{2} \delta_{a-b}$ for $\lambda$. But there are many other possibilities, since if $X$ is a symmetric random vector, and $X$ and $\Theta$ are independent, then $X \Theta \stackrel{d}{=} X|\Theta|$. Thus the measure $\lambda$ is not uniquely 
determined and condition (B) holds for every $\lambda_{p q}, p, q \in[0,1 / 2]$, where

$$
\lambda_{p q}:=p \delta_{a+b}+\left(\frac{1}{2}-p\right) \delta_{-a-b}+q \delta_{a-b}+\left(\frac{1}{2}-q\right) \delta_{b-a} .
$$

It is easy to see that the set $K\left(\delta_{a}, \delta_{b}\right)=\left\{\lambda_{p q}: p, q \in[0,1 / 2]\right\}$ is closed and convex. This property turns out to be general.

In [3, 10-13] Kucharczak, Urbanik and Vol'kovich considered a very similar problem. They studied the properties of weakly stable random variables and measures, where a random variable $X \geq 0$ with distribution $\mu$ on $[0, \infty)$ is said to be weakly stable if for any $a, b \in \mathbb{R}_{+}$there exists a nonnegative random variable $Q$ with distribution $\lambda$ such that

$$
T_{a} \mu * T_{b} \mu=\mu \circ \lambda .
$$

From now on we will say that a distribution $\mu$ for which (C) holds is $\mathbb{R}_{+^{-}}$ weakly stable, and that $\mu$ is weakly stable when (B) is satisfied. The next example shows that these two conditions are not equivalent.

EXAmple 2. Assume that a random vector $X$ has a symmetric $\alpha$-stable distribution $\mu$ with $\alpha \in(0,2]$. This means that for every $a, b \in \mathbb{R}$ we have $a X+b X^{\prime} \stackrel{d}{=} c X$, where $c^{\alpha}=|a|^{\alpha}+|b|^{\alpha}$, so condition (B) holds for $\lambda=\delta_{c}$. It is easy to see that the opposite implication also holds, i.e. if for every $a, b \in \mathbb{R}$ there exists a Dirac measure satisfying condition (B), then $\mu$ is symmetric stable. This is a little different from the usual condition, where the assumption

$$
\forall a, b>0 \exists c>0, \quad a X+b X^{\prime} \stackrel{d}{=} c X .
$$

is equivalent to $X$ having a strictly stable distribution. Thus, a strictly stable distribution is $\mathbb{R}_{+}$-weakly stable, but it may not be weakly stable. A symmetric stable distribution is both $\mathbb{R}_{+}$-weakly stable and weakly stable.

EXAMPLE 3. Consider the random vector $X_{k, n}=\left(U_{1}, \ldots, U_{k}\right)$ for $k \leq n$ which is the $k$-dimensional projection of $U^{n}=\left(U_{1}, \ldots, U_{n}\right)$ with the uniform distribution on the unit sphere $S_{n-1} \subset \mathbb{R}^{n}$. The distribution $\mu_{k, n}$ of $X_{k, n}$ for $k<n$ is absolutely continuous with respect to the Lebesgue measure with density

$$
f\left(x_{1}, \ldots, x_{k}\right)=c(n, k)\left(1-\sum_{i=1}^{k} x_{i}^{2}\right)_{+}^{(n-k) / 2-1},
$$

where $c(n, k)$ is a normalizing constant. The set $\mathcal{M}\left(\mu_{n, n}\right)$ is well known, being the set of all rotationally invariant distributions on $\mathbb{R}^{n}$. The set $\mathcal{M}\left(\mu_{k, n}\right)$ is a convex and closed subset of $\mathcal{M}\left(\mu_{k, k}\right)$. If $n=k+2$, then $\mu_{k, n}$ is the uniform distribution on the unit ball $B_{k} \subset \mathbb{R}^{k}$. In particular, $\mathcal{M}\left(\mu_{1,3}\right)$ is the set of symmetric unimodal probability measures on $\mathbb{R}$. 
In order to show that all these classes are also closed under convolution, we need to use the following characterization:

$\mu$ is rotationally invariant on $\mathbb{R}^{k}$

$\Leftrightarrow \widehat{\mu}(\xi)$ depends only on $\|\xi\|_{2}=\left(\left|\xi_{1}\right|^{2}+\cdots+\left|\xi_{k}\right|^{2}\right)^{1 / 2}$, i.e. $\widehat{\mu}(\xi)=\varphi\left(\|\xi\|_{2}\right)$ for some function $\varphi$

$\Leftrightarrow \mu$ is the distribution of $U^{n} \Theta$, where $\Theta \geq 0$ is independent of $U^{n}$.

Now, let $\nu_{1}, \nu_{2} \in \mathcal{M}\left(\mu_{k, n}\right)$. This means that there exist independent rotationally invariant random vectors $X_{1}$ and $X_{2}$ on $\mathbb{R}^{n}$ such that $\nu_{1}$ and $\nu_{2}$ are the distributions of the $k$-dimensional projections of $X_{1}$ and $X_{2}$. For every $a, b \in \mathbb{R}$, the random vector $a X_{1}+b X_{2}$ is also rotationally invariant on $\mathbb{R}^{n}$ since

$$
\begin{aligned}
& \mathbf{E} \exp \left\{i\left\langle a X_{1}, \xi\right\rangle+i\left\langle b X_{2}, \xi\right\rangle\right\} \\
& \quad=\mathbf{E} \exp \left\{i\left\langle a X_{1}, \xi\right\rangle\right\} \mathbf{E} \exp \left\{i\left\langle b X_{2}, \xi\right\rangle\right\}=f_{1}\left(|a|\|\xi\|_{2}\right) f_{2}\left(|b|\|\xi\|_{2}\right),
\end{aligned}
$$

so the right hand side is a function depending only on $\|\xi\|_{2}(a, b$ are just some parameters here). This means that there exists a random variable $Q=Q_{a, b}$ such that $a X_{1}+b X_{2} \stackrel{d}{=} U^{n} Q$. It is easy to see now that $T_{a} \nu_{1}+T_{b} \nu_{2}$ is the distribution of a $k$-dimensional projection of $U^{n} Q$, which was to be shown. It is interesting that the variable $Q_{a, b}$ for the measure $\mu_{k, n}$ does not depend on $k$; in fact $Q_{a, b}$ has the same distribution as $\left\|a X_{1}+b X_{2}\right\|_{2}$.

\section{Conditions (A) and (B) are equivalent}

Lemma 1. Assume that a measure $\mu$ has property (B). Then, for any discrete measures $\nu_{1}=\sum_{i} p_{i} \delta_{a_{i}}$ and $\nu_{2}=\sum_{i} q_{i} \delta_{b_{i}}$, the measure $\left(\mu \circ \nu_{1}\right) *$ $\left(\mu \circ \nu_{2}\right)$ belongs to $\mathcal{M}(\mu)$.

Proof. Let $\lambda_{i j}$ be such that $T_{a_{i}} \mu * T_{b_{j}} \mu=\mu \circ \lambda_{i j}$. Then

$$
\left(\mu \circ \nu_{1}\right) *\left(\mu \circ \nu_{2}\right)=\sum_{i, j} p_{i} q_{j} T_{a_{i}} \mu * T_{b_{j}} \mu=\sum_{i, j} p_{i} q_{j} \mu \circ \lambda_{i j}=\mu \circ\left(\sum_{i, j} p_{i} q_{j} \lambda_{i j}\right) .
$$

LeMma 2. Let $\mu \neq \delta_{0}$ be a probability measure on a separable Banach space $\mathbb{E}$ and let $\mathcal{A} \subset \mathcal{P}$. If the set $\mathcal{B}=\{\mu \circ \lambda: \lambda \in \mathcal{A}\}$ is tight, then so is $\mathcal{A}$.

Proof. Let $\mu=\mathcal{L}(X)$ and $\lambda=\mathcal{L}\left(Q_{\lambda}\right)$ for $X$ and $Q_{\lambda}$ independent, $\lambda \in \mathcal{A}$. Let $\varepsilon>0$. Since $\mathcal{B}$ is tight there exists a compact set $L \subset \mathbb{E}$ such that

$$
\mathbf{P}\left(Q_{\lambda} X \in L\right) \geq 1-\varepsilon \mathbf{P}(X \neq 0) .
$$

Put $L_{n}=[-1 / n, 1 / n] \cdot L=\{s x: s \in[-1 / n, 1 / n], x \in L\}$. Since $L$ is bounded we have

$$
\liminf _{n \rightarrow \infty} \mathbf{P}\left(X \notin L_{n}\right) \geq \mathbf{P}(X \neq 0) .
$$


Choose $n$ such that $\mathbf{P}\left(X \notin L_{n}\right) \geq \mathbf{P}(X \neq 0) / 2$. Then

$$
\begin{aligned}
\varepsilon \mathbf{P}(X \neq 0) & \geq \mathbf{P}\left(Q_{\lambda} X \notin L\right) \geq \mathbf{P}\left(\left|Q_{\lambda}\right|>n, X \notin L_{n}\right) \\
& =\mathbf{P}\left(\left|Q_{\lambda}\right|>n\right) \mathbf{P}\left(X \notin L_{n}\right) \geq \mathbf{P}\left(\left|Q_{\lambda}\right|>n\right) \mathbf{P}(X \neq 0) / 2,
\end{aligned}
$$

so that $\mathbf{P}\left(\left|Q_{\lambda}\right|>n\right) \leq 2 \varepsilon$ for all $\lambda \in \mathcal{A}$. This implies tightness of $\mathcal{A}$.

Lemma 3. The set $\mathcal{M}(\mu)$ is closed in the topology of weak convergence and the set of extreme points of $\mathcal{M}(\mu)$ is $\left\{T_{a} \mu: a \in \mathbb{R}\right\}$.

Proof. If $\mu=\delta_{0}$ then the assertion follows immediately, so we assume that $\mu \neq \delta_{0}$. Assume that $\mu \circ \lambda_{n} \Rightarrow \nu$. Then the set $\left\{\mu \circ \lambda_{n}: n \in \mathbb{N}\right\}$ is tight, and, by Lemma 2 the set $\left\{\lambda_{n}: n \in \mathbb{N}\right\}$ is also tight. Thus it contains a subsequence $\lambda_{n_{k}}$ converging weakly to a probability measure $\lambda$ on $\mathbb{R}$. Since the function $\widehat{\mu}(t)$ is bounded and continuous, we obtain

$$
\int \widehat{\mu}(t s) \lambda_{n_{k}}(d s) \rightarrow \int \widehat{\mu}(t s) \lambda(d s) .
$$

On the other hand, we have

$$
\int \widehat{\mu}(t s) \lambda_{n}(d s) \rightarrow \widehat{\nu}(t)
$$

This means that $\nu=\mu \circ \lambda$ and consequently $\nu \in \mathcal{M}(\mu)$.

If $a=0$, then $T_{a} \mu=\delta_{0}$ and it is easy to check that $\delta_{0}$ is an extreme point in $\mathcal{M}(\mu)$. Assume that for some $a \in \mathbb{R}, a \neq 0$, there exist $\lambda_{1}, \lambda_{2} \in \mathcal{P}$ and $p \in(0,1)$ such that

$$
T_{a} \mu=p \mu \circ \lambda_{1}+(1-p) \mu \circ \lambda_{2}=\mu \circ\left(p \lambda_{1}+(1-p) \lambda_{2}\right) .
$$

This means that $a X \stackrel{d}{=} X \Theta$ for some random variable $\Theta$ independent of $X$ with distribution $p \lambda_{1}+(1-p) \lambda_{2}$. The result of Mazurkiewicz (see [5]) implies that $\mathbf{P}\{\Theta=a\}=1$ if the distribution of $X$ is not symmetric, and $\mathbf{P}\{|\Theta|=|a|\}=1$ otherwise. In the first situation we would have

$$
\delta_{a}=p \lambda_{1}+(1-p) \lambda_{2},
$$

so $\lambda_{1}=\lambda_{2}=\delta_{a}$ since $\delta_{a}$ is an extreme point in $\mathcal{P}$. If $X$ has symmetric distribution we obtain

$$
\begin{aligned}
\delta_{|a|}(A) & =p \lambda_{1}(A)+(1-p) \lambda_{2}(A)+p \lambda_{1}(-A)+(1-p) \lambda_{2}(-A) \\
& =: p\left|\lambda_{1}\right|(A)+(1-p)\left|\lambda_{2}\right|(A)
\end{aligned}
$$

for every Borel set $A \subset(0, \infty)$. Since $\delta_{|a|}$ is an extreme point in $\mathcal{P}_{+}$, we have $\delta_{|a|}=\left|\lambda_{1}\right|=\left|\lambda_{2}\right|$. Now, it is enough to notice that for a symmetric distribution $\mu$, the equality $\mu \circ \lambda=\mu \circ|\lambda|$ holds for every probability measure $\lambda$. Consequently, we obtain

$$
T_{a} \mu=\mu \circ\left|\lambda_{1}\right|=\mu \circ \lambda_{1}=\mu \circ\left|\lambda_{2}\right|=\mu \circ \lambda_{2} .
$$

The above reasoning works for $\mu \in \mathcal{P}$. For $\mu \in \mathcal{P}(\mathbb{E})$ the following two situations are possible. If $\mu$ is nonsymmetric then one can choose $\xi \in \mathbb{E}^{*}$ such that $\xi(X)$ is nonsymmetric and use the result of Mazurkiewicz as before. If 
$\mu$ is symmetric then there exists $\xi \in \mathbb{E}^{*}$ such that $\xi(X) \not \equiv 0$ since $\mu \neq \delta_{0}$, so that $\delta_{|a|}=\left|\lambda_{1}\right|=\left|\lambda_{2}\right|$, as before. The rest of the reasoning does not need any change.

Assume now that the probability measure $\nu$ is an extreme point of $\mathcal{M}(\mu)$. Then there exists a probability measure $\lambda$ such that $\nu=\mu \circ \lambda$. If $\lambda \neq \delta_{a}$ for any $a \in \mathbb{R}$ then we could divide $\mathbb{R}$ into two Borel sets $A$ and $A^{\prime}=\mathbb{R} \backslash A$ such that $\lambda(A)=\alpha \in(0,1)$. Then

$$
\mu=\alpha \mu \circ\left(\left.\alpha^{-1} \lambda\right|_{A}\right)+(1-\alpha) \mu \circ\left(\left.(1-\alpha)^{-1} \lambda\right|_{A^{\prime}}\right),
$$

in contradiction with the assumption that $\nu$ is extremal.

Lemma 4. Assume that for a probability measure $\mu \neq \delta_{0}$ and some $\nu_{1}, \nu_{2} \in \mathcal{P}$ the set

$$
K_{\mu}\left(\nu_{1}, \nu_{2}\right):=\left\{\lambda:\left(\mu \circ \nu_{1}\right) *\left(\mu \circ \nu_{2}\right)=\mu \circ \lambda\right\}
$$

is not empty. Then it is convex and weakly compact.

Proof. Notice that

$$
\left\{\left(\mu \circ \nu_{1}\right) *\left(\mu \circ \nu_{2}\right)\right\}=\left\{\mu \circ \lambda: \lambda \in K_{\mu}\left(\nu_{1}, \nu_{2}\right)\right\},
$$

and the set $\left\{\left(\mu \circ \nu_{1}\right) *\left(\mu \circ \nu_{2}\right)\right\}$ contains only one point. Then the weak compactness of $K_{\mu}\left(\nu_{1}, \nu_{2}\right)$ follows from Lemma 2 . The convexity is trivial.

Lemma 5. Assume that $\mu \neq \delta_{0}$ is a probability measure and $K_{\mu}\left(\nu_{n}^{1}, \nu_{n}^{2}\right)$ $\neq \emptyset$ for every $n \in \mathbb{N}$, where $\nu_{n}^{1} \rightarrow \nu_{1}$ weakly, $\nu_{n}^{2} \rightarrow \nu_{2}$ weakly, and $\nu_{n}^{i}, \nu_{i} \in \mathcal{P}$. Then $K_{\mu}\left(\nu_{1}, \nu_{2}\right) \neq \emptyset$.

$$
\begin{aligned}
& \text { Proof. Let } \mathcal{A}=\bigcup_{n=1}^{\infty} K_{\mu}\left(\nu_{n}^{1}, \nu_{n}^{2}\right) \text { and } \\
& \qquad \mathcal{B}=\{\mu \circ \lambda: \lambda \in \mathcal{A}\}=\left\{\left(\mu \circ \nu_{n}^{1}\right) *\left(\mu \circ \nu_{n}^{2}\right): n \in \mathbb{N}\right\} .
\end{aligned}
$$

Since $\mathcal{B}$ is tight, so is $\mathcal{A}$ by Lemma 2 . Choosing now $\lambda_{n} \in K_{\mu}\left(\nu_{n}^{1}, \nu_{n}^{2}\right)$ for every $n \in \mathbb{N}$, we can find a subsequence $\lambda_{n_{k}}$ converging weakly to a probability measure $\lambda$. Since

$$
\left(\mu \circ \nu_{n_{k}}^{1}\right) *\left(\mu \circ \nu_{n_{k}}^{2}\right)=\mu \circ \lambda_{n_{k}},
$$

we also have

$$
\left(\mu \circ \nu_{1}\right) *\left(\mu \circ \nu_{2}\right)=\mu \circ \lambda
$$

and consequently $\lambda \in K_{\mu}\left(\nu_{1}, \nu_{2}\right) \neq \emptyset$.

THEOREM 1. For every probability distribution $\mu$ properties (A) and (B) are equivalent.

Proof. The implication $(\mathrm{A}) \Rightarrow(\mathrm{B})$ is trivial. Assume that $\mu \neq \delta_{0}$ and (B) holds. This means that $K_{\mu}\left(\delta_{a}, \delta_{b}\right) \neq \emptyset$ for any $a, b \in \mathbb{R}$. It follows from Lemma 1 that $K_{\mu}\left(\nu_{1}, \nu_{2}\right) \neq \emptyset$ for any discrete measures $\nu_{1}, \nu_{2}$. Let now $\lambda_{1}, \lambda_{2} \in \mathcal{P}$. We can find two sequences of discrete measures $\nu_{1, n}$ and $\nu_{2, n}$ converging weakly to $\lambda_{1}$ and $\lambda_{2}$ respectively. Since $K_{\mu}\left(\nu_{1, n}, \nu_{2, n}\right) \neq \emptyset$ for every $n \in \mathbb{N}$, Lemma 5 shows that also $K_{\mu}\left(\lambda_{1}, \lambda_{2}\right) \neq \emptyset$, which implies (A). 
Proposition 1. Let $X=\left(X_{1}, \ldots, X_{n}\right)$ be a symmetric $\alpha$-stable random vector, and let $\Theta$ be a random variable independent of $X$. Then $Y=X \Theta$ is weakly stable iff $|\Theta|^{\alpha}$ is $\mathbb{R}_{+}$-weakly stable.

Proof. Notice that

$$
a X \Theta+b X^{\prime} \Theta^{\prime} \stackrel{d}{=}\left(|a \Theta|^{\alpha}+\left|b \Theta^{\prime}\right|^{\alpha}\right)^{1 / \alpha} X,
$$

where $X^{\prime}, \Theta^{\prime}$ are independent copies of $X, \Theta$ such that $X, X^{\prime}, \Theta, \Theta^{\prime}$ are independent. Assume that $Y$ is weakly stable. Since $X \Theta \stackrel{d}{=} X|\Theta|$ we obtain

$$
\left(|a \Theta|^{\alpha}+\left|b \Theta^{\prime}\right|^{\alpha}\right)^{1 / \alpha} X \stackrel{d}{=} X \cdot|\Theta| \cdot Q
$$

for some random variable $Q$. Without loss of generality we can assume that $Q \geq 0$. A symmetric stable distribution is cancellable (see [3, Prop. 1.1]), thus we obtain

$$
|a|^{\alpha}|\Theta|^{\alpha}+|b|^{\alpha}\left|\Theta^{\prime}\right|^{\alpha} \stackrel{d}{=}|\Theta|^{\alpha} Q^{\alpha}
$$

This implies that $|\Theta|^{\alpha}$ is $\mathbb{R}_{+}$-weakly stable. The converse is trivial.

3. Symmetrizations of mixing measures are uniquely determined. Assume that a measure $\mu \neq \delta_{0}$ on $\mathbb{R}$ is weakly stable. We have seen before that $K_{\mu}\left(\nu_{1}, \nu_{2}\right)$ is a nonempty convex and weakly compact set in $\mathcal{P}$ for all $\nu_{1}, \nu_{2} \in \mathcal{P}$. In this section we discuss further properties of $K_{\mu}\left(\nu_{1}, \nu_{2}\right)$.

For a weakly stable measure $\mu$ we define

$$
\Phi(\mu)=\{\widehat{\nu}: \nu=\mu \circ \lambda, \lambda \in \mathcal{P}\},
$$

and let $L(\mu)$ denote the complex linear space generated by $\Phi(\mu)$. Weak stability of $\mu$ implies that for any $f, g \in L(\mu)$ we have $f g, \bar{f} \in L(\mu)$. Since $\mu \circ \delta_{0}=\delta_{0}$ the space $L(\mu)$ contains the constants.

We denote by $\overline{\mathbb{R}}=\mathbb{R} \cup\{\Delta\}$ the one-point compactification of the real line, and by $\overline{\mathbb{R}}_{+}=\mathbb{R}_{+} \cup\{\infty\}$ the one-point compactification of the nonnegative half-line. Let $C(Y)$ denote the space of continuous real functions on the topological space $Y$. Then $C\left(\overline{\mathbb{R}}_{+}\right)$can be identified with the set of even (symmetric) functions from $C(\overline{\mathbb{R}})$.

Now, for a probability measure $\mu$, we define

$$
A(\mu)=\left\{f \in L(\mu): f=\bar{f}, \lim _{x \rightarrow \infty} f(x)=\lim _{x \rightarrow-\infty} f(x)\right\} .
$$

If $\mu$ is weakly stable then $A(\mu)$ is an algebra (over the reals).

Lemma 6. If a probability measure $\mu$ on $\mathbb{R}$ is not symmetric, then the set $A(\mu)$ separates points of $\overline{\mathbb{R}}$.

Proof. Let $\gamma$ be a symmetric Cauchy distribution with Fourier transform $\widehat{\gamma}(t)=e^{-|t|}$. For every $c \in \mathbb{R}$, we define

$$
h_{c}(t)=\left(\mu \circ\left(\gamma * \delta_{c}\right)\right)^{\wedge}(t) \in \Phi(\mu) .
$$


First we show that there exists $a \in \mathbb{R}$ such that $\Im m\left(h_{a}\right) \neq 0$. Assume the opposite, i.e. $\Im m\left(h_{c}\right) \equiv 0$ for every $c \in \mathbb{R}$. This means that

$$
\Im m\left(h_{c}(t)\right)=\int_{-\infty}^{\infty} e^{-|t x|} \sin (c t x) \mu(d x)=0
$$

for all $c, t \in \mathbb{R}$. Substituting $u=c t$, we obtain

$$
\int_{-\infty}^{\infty} e^{-|u x| /|c|} \sin (u x) \mu(d x)=0
$$

for $u \in \mathbb{R}$ and $c \neq 0$. This implies that

$$
\lim _{c \rightarrow \infty} \int_{-\infty}^{\infty} e^{-|u x| /|c|} \sin (u x) \mu(d x)=\int_{-\infty}^{\infty} \sin (u x) \mu(d x)=0,
$$

which means that the characteristic function $\widehat{\mu}$ is real, which contradicts our assumption.

Now let $a, t_{0} \in \mathbb{R}$ be such that $\Im m\left(h_{a}\left(t_{0}\right)\right) \neq 0$. For every $s \neq 0$, we define

$$
g_{s}(t)=\Im m\left(h_{a}\left(\frac{t \cdot t_{0}}{s}\right)\right) .
$$

It is easy to see that $g_{s}(t) \in A(\mu)$, and $g_{s}(t)=-g_{s}(-t)$. We can now see that for every $r \in \mathbb{R}, r \neq 0$, the function $g_{r}(t)$ separates the points $r$ and $-r$ since $g_{r}(r)=h_{a}\left(t_{0}\right) \neq g_{r}(-r)$. To finish the proof, it is enough to notice that the function

$$
h_{0}(t)=\int_{-\infty}^{\infty} e^{-|t x|} \mu(d x)
$$

separates points $t_{1}, t_{2} \in \mathbb{R}$ if $\left|t_{1}\right| \neq\left|t_{2}\right|$, including the case $t_{i}=\Delta$.

Lemma 7. If a probability measure $\mu$ on $\mathbb{R}$ is symmetric and $\mu \neq \delta_{0}$, then $A(\mu)$ separates points of $\overline{\mathbb{R}}_{+}$.

Proof. It is enough to notice that the function $h_{0}(t)=\int e^{-|t x|} \mu(d x)$ separates points of $\overline{\mathbb{R}}_{+}$.

THEOREM 2. If a weakly stable measure $\mu \neq \delta_{0}$ on $\mathbb{R}$ is not symmetric, then for any $\nu_{1}, \nu_{2} \in \mathcal{P}$ the set $K_{\mu}\left(\nu_{1}, \nu_{2}\right)$ contains only one measure.

Proof. Assume that $\lambda_{1}, \lambda_{2} \in K_{\mu}\left(\nu_{1}, \nu_{2}\right)$. This means that $\mu \circ \lambda_{1}=\mu \circ \lambda_{2}$, and consequently, for every $\lambda \in \mathcal{P}$,

$$
(\mu \circ \lambda) \circ \lambda_{1}=(\mu \circ \lambda) \circ \lambda_{2} .
$$

Hence, for every $\lambda \in \mathcal{P}$,

$$
\int_{-\infty}^{\infty}(\mu \circ \lambda)^{\wedge}(t x) \lambda_{1}(d x)=\int_{-\infty}^{\infty}(\mu \circ \lambda)^{\wedge}(t x) \lambda_{2}(d x) .
$$


This implies that for every $f \in A(\mu)$,

$$
\int_{-\infty}^{\infty} f(x) \lambda_{1}(d x)=\int_{-\infty}^{\infty} f(x) \lambda_{2}(d x)
$$

From Lemma 6 we know that the algebra $A(\mu)$ separates points of $\overline{\mathbb{R}}$, so by the Stone-Weierstrass Theorem (see Theorem $4 \mathrm{E}$ in $[4]$ ), it is dense in $C(\overline{\mathbb{R}})$ in the topology of uniform convergence. This means that $(*)$ holds for every $f \in C(\overline{\mathbb{R}})$, and consequently $\lambda_{1}=\lambda_{2}$.

Let $\tau=\frac{1}{2} \delta_{1}+\frac{1}{2} \delta_{-1}$. The symmetrization of a measure $\lambda \in \mathcal{P}$ is defined to be the measure $\lambda \circ \tau$. Notice that $\lambda$ is symmetric if and only if $\lambda=\lambda \circ \tau$.

THEOREM 3. If a weakly stable measure $\mu \neq \delta_{0}$ on $\mathbb{R}$ is symmetric and $\nu_{1}, \nu_{2} \in \mathcal{P}$, then

$$
\lambda_{1}, \lambda_{2} \in K_{\mu}\left(\nu_{1}, \nu_{2}\right) \Rightarrow \lambda_{1} \circ \tau=\lambda_{2} \circ \tau .
$$

If $\lambda_{1} \circ \tau=\lambda_{2} \circ \tau$ and $\lambda_{1} \in K_{\mu}\left(\nu_{1}, \nu_{2}\right)$ then $\lambda_{2} \in K_{\mu}\left(\nu_{1}, \nu_{2}\right)$.

Proof. The second implication is trivial because for every symmetric measure $\mu$ we have $\mu \circ \lambda=\mu \circ(\lambda \circ \tau)$. To prove the first implication assume that $\lambda_{1}, \lambda_{2} \in K_{\mu}\left(\nu_{1}, \nu_{2}\right)$. This implies that $\mu \circ \lambda_{1}=\mu \circ \lambda_{2}$, and consequently $(\mu \circ \lambda) \circ \lambda_{1}=(\mu \circ \lambda) \circ \lambda_{2}$ for every $\lambda \in \mathcal{P}$. This means that for every even function $f \in A(\mu)$ the following equality holds:

$$
\int_{0}^{\infty} f(x)\left(\tau \circ \lambda_{1}\right)(d x)=\int_{0}^{\infty} f(x)\left(\tau \circ \lambda_{2}\right)(d x) .
$$

It follows from the proof of Lemma 7 that the even functions from $A(\mu)$ separate points in $\overline{\mathbb{R}}_{+}$. Applying the Stone-Weierstrass Theorem again we conclude that the set of even functions from $A(\mu)$ is dense in $C\left(\overline{\mathbb{R}}_{+}\right)$in the topology of uniform convergence. This means that $(* *)$ holds for every $f \in C\left(\overline{\mathbb{R}}_{+}\right)$, so the measures $\tau \circ \lambda_{1}$ and $\tau \circ \lambda_{2}$ coincide on $\mathbb{R}_{+}$, and, by symmetry, also on $\mathbb{R}$.

REMARK 1. Notice that it follows from the proofs of Theorems 2 and 3 that weakly stable distributions are reducible in the sense that:

- If $X, Y, Z$ are independent real random variables and $X$ is nonsymmetric and weakly stable then the equality $X Y \stackrel{d}{=} X Z$ implies $\mathcal{L}(Y)=\mathcal{L}(Z)$.

- If $X, Y, Z$ are independent, $Y, Z$ are real, and $X$ is a nonsymmetric weakly stable random vector taking values in a separable Banach space $\mathbb{E}$, then $X Y \stackrel{d}{=} X Z$ implies $\mathcal{L}(Y)=\mathcal{L}(Z)$. To see this, apply the previous remark to the random variable $\xi(X)$, where $\xi \in \mathbb{E}^{*}$ is such that $\xi(X)$ is not symmetric.

- If $X, Y, Z$ are independent, $Y, Z$ take values in $\mathbb{E}$, and $X$ is a nonsymmetric weakly stable real random variable, then $X Y \stackrel{d}{=} X Z$ implies 
$\mathcal{L}(Y)=\mathcal{L}(Z)$. To see this, note that it suffices to prove $\xi(Y) \stackrel{d}{=} \xi(Z)$ for all $\xi \in \mathbb{E}^{*}$.

- If $X, Y, Z$ are independent and $X \not \equiv 0$ is symmetric weakly stable, then $X Y \stackrel{d}{=} X Z$ implies $\mathcal{L}(Y) \circ \tau=\mathcal{L}(Z) \circ \tau$.

REMARK 2. Notice that if $\mu$ is weakly stable then so is $\mu \circ \tau$. Indeed, if $T_{a} \mu * T_{b} \mu=\nu_{1} \circ \mu$ and $T_{a} \mu * T_{-b} \mu=\nu_{2} \circ \tau$ then

$$
T_{a}(\mu \circ \tau) * T_{b}(\mu \circ \tau)=\left(\frac{1}{2} \nu_{1}+\frac{1}{2} \nu_{2}\right) \circ(\mu \circ \tau) .
$$

\section{Some general properties of weakly stable distributions}

Lemma 8. If a measure $\mu$ on $\mathbb{R}$ is weakly stable then $\mu(\{0\})=0$ or 1 .

Proof. Let $X$ be a weakly stable variable such that $\mathcal{L}(X)=\mu, \mathbf{P}\{X=0\}$ $=p<1$, and let $X^{\prime}$ be its independent copy. We define the random variable $Y$ with distribution $\mathcal{L}(X \mid X \neq 0)$ and $Y^{\prime}$ its independent copy. The random variable $Y / Y^{\prime}$ has at most countably many atoms, so there exists $a \in \mathbb{R}$, $a \neq 0$, such that $\mathbf{P}\left\{Y=a Y^{\prime}\right\}=0$. Now let $\Theta$ be the random variable independent of $X$ such that

$$
X-a X^{\prime} \stackrel{d}{=} X \Theta
$$

Then we have

$p \leq \mathbf{P}\{X \Theta=0\}=\mathbf{P}\left\{X-a X^{\prime}=0\right\}=p^{2}+(1-p)^{2} \mathbf{P}\left\{Y-a Y^{\prime}=0\right\}=p^{2}$.

This holds only if $p=0$, which ends the proof.

Lemma 9. Assume that a weakly stable probability measure $\mu \neq \delta_{0}$ on $\mathbb{R}$ has at least one atom. Then the discrete part of $\mu$ (normalized to be a probability measure) is also weakly stable.

Proof. Let $\mu=\alpha \mu_{1}+(1-\alpha) \mu_{2}, \alpha \in(0,1)$, where $\alpha \mu_{1}$ is the discrete part of $\mu, \mu_{1}(\mathbb{R})=1$, and $\mu_{2}$ is such that $\mu_{2}(\mathbb{R})=1$ and $\mu_{2}(\{x\})=0$ for every $x \in \mathbb{R}$. If $\mu$ is weakly stable, then for every $a \in \mathbb{R}$ there exists a probability measure $\lambda$ such that $\mu * T_{a} \mu=\mu \circ \lambda$. Now we have

$$
\begin{aligned}
\mu * T_{a} \mu= & \alpha^{2} \mu_{1} * T_{a} \mu_{1}+\alpha(1-\alpha) \mu_{1} * T_{a} \mu_{2} \\
& +\alpha(1-\alpha) \mu_{2} * T_{a} \mu_{1}+(1-\alpha)^{2} \mu_{2} * T_{a} \mu_{2} .
\end{aligned}
$$

Clearly for $a \neq 0$ the discrete part of $\mu * T_{a} \mu$ is equal to $\alpha^{2} \mu_{1} * T_{a} \mu_{1}$. On the other hand, we have

$$
\mu \circ \lambda=(1-\beta) \mu \circ \lambda_{2}+\alpha \beta \mu_{1} \circ \lambda_{1}+(1-\alpha) \beta \mu_{2} \circ \lambda_{1},
$$

where $\lambda_{1}(\mathbb{R})=\lambda_{2}(\mathbb{R})=1, \lambda_{1}$ is a discrete measure, $\lambda_{2}(\{x\})=0$ for every $x \in \mathbb{R}$ and $\lambda=\beta \lambda_{1}+(1-\beta) \lambda_{2}$. 
Let $S=\left\{a \in \mathbb{R}: \mu * T_{a} \mu(\{0\})=0\right\}$. If $a \in S, a \neq 0$, then $\lambda(\{0\})=0$ and $\mu \circ \lambda_{2}(\{x\})=\beta \mu_{2} \circ \lambda_{1}(\{x\})=0$ for every $x \in \mathbb{R}$, so

$$
\alpha^{2} \mu_{1} * T_{a} \mu_{1}=\alpha \beta \mu_{1} \circ \lambda_{1} \text {. }
$$

This means that $\alpha=\beta$ and $\mu_{1} * T_{a} \mu_{1}=\mu_{1} \circ \lambda_{1}$.

If $a \notin S$ then there exists a sequence $a_{n} \in S \backslash\{0\}, n \in \mathbb{N}$, such that $\lim _{n} a_{n}=a$. Then $\mu * T_{a_{n}} \mu \Rightarrow \mu * T_{a} \mu$ and $\mu_{1} * T_{a_{n}} \mu_{1} \Rightarrow \mu_{1} * T_{a} \mu_{1}$. For every $n \in \mathbb{N}$ there exists $\lambda_{n}$ such that $\mu_{1} * T_{a_{n}} \mu_{1}=\mu_{1} \circ \lambda_{n}$, i.e. $\lambda_{n} \in K_{\mu_{1}}\left(\delta_{1}, \delta_{a_{n}}\right)$. In view of Lemma 5 there exists $\lambda \in K_{\mu_{1}}\left(\delta_{1}, \delta_{a}\right)$, which ends the proof.

THeOREM 4. Assume that a random vector $X$ taking values in a separable Banach space $\mathbb{E}$ and having distribution $\mu$ is such that $\mathbf{E}\|X\|<\infty$ and $\mathbf{E} X=a \neq 0$. Then $\mu$ is weakly stable if and only if $\mu=\delta_{a}$.

Proof. Assume first that $\mathbb{E}=\mathbb{R}$. If $\mu=\delta_{a}$ for some $a \neq 0$, then it is weakly stable. Conversely, let $\mu$ be weakly stable and $\mathbf{E} X=a \neq 0$. Let $X_{1}, X_{2}, \ldots$ be a sequence of i.i.d. random variables with distribution $\mu$. The Weak Law of Large Numbers implies that

$$
\frac{1}{n} \sum_{k=1}^{n} X_{k} \rightarrow a
$$

weakly as $n \rightarrow \infty$. The measure $\mu$ is weakly stable, thus for every $n \in \mathbb{N}$ there exists a measure $\nu_{n}$ such that

$$
\mu_{n}=\mathcal{L}\left(\frac{1}{n} \sum_{k=1}^{n} X_{k}\right)=\left(T_{1 / n} \mu\right)^{* n}=\mu \circ \nu_{n} .
$$

Since $\mu_{n} \Rightarrow \delta_{a}$, it follows from Lemma 2 that the family $\left\{\nu_{n}\right\}$ is tight and it contains a sequence $\nu_{n_{k}}$ such that $\nu_{n_{k}} \Rightarrow \nu$ for some probability measure $\nu$. Now, we obtain

$$
\delta_{a}=\lim _{n \rightarrow \infty} \mu_{n}=\lim _{k \rightarrow \infty} \mu \circ \nu_{n_{k}}=\mu \circ \nu .
$$

Since $a \neq 0$ the last equality is possible only if $\mu=\delta_{x}$ and $\nu=\delta_{y}$ for some $x, y \in \mathbb{R}$ with $x y=a$. Since $\mathbf{E} X=a$, we conclude that $\mu=\delta_{a}$.

If $X$ is a random vector in a separable Banach space $\mathbb{E}$ with $\mathbf{E} X=$ $a \neq 0$ then the previous considerations yield $\mathbf{P}\{\xi(X)=\xi(a)\}=1$ for each $\xi \in \mathbb{E}^{*}$ with $\xi(a) \neq 0$. Such $\xi$ 's form a dense subset in $\mathbb{E}^{*}$. Consequently, $\mathbf{P}\{X=a\}=1$.

THEOREM 5. Assume that for a weakly stable measure $\mu \neq \delta_{0}$ on a separable Banach space $\mathbb{E}$ there exists $\varepsilon \in(0,1]$ such that for every $\xi \in \mathbb{E}^{*}$ and every $p \in(0, \varepsilon)$,

$$
\int_{\mathbb{E}}|\xi(x)|^{p} \mu(d x)<\infty .
$$


Then there exists $\alpha_{0} \in[\varepsilon, 2]$ such that $\mathcal{M}(\mu)$ contains a strictly $\alpha$-stable measure for every $\alpha \in\left(0, \alpha_{0}\right)$.

Proof. Let $p \in(0, \varepsilon)$. Since $\mathcal{M}(\mu)$ is closed under scale mixing, $\mu \circ m_{n}$ $\in \mathcal{M}(\mu)$ for every $n \in \mathbb{N}$, where

$$
m_{n}(d x)=c(n) x^{-p-1} \mathbf{1}_{(1 / n, \infty)} d x, \quad c(n)=p n^{-p} .
$$

As $\mathcal{M}(\mu)$ is also closed under convolution and under taking convex linear combinations, and weakly closed, for every $n \in \mathbb{N}$ we have

$$
\nu_{n}=\exp \left\{c(n)^{-1}\left(\mu \circ m_{n}\right)\right\} \in \mathcal{M}(\mu),
$$

where $\exp (\kappa):=e^{-\kappa(\mathbb{E})} \sum_{k=0}^{\infty} \kappa^{* k} / k$ ! for every finite measure $\kappa$ on $\mathbb{E}$. Notice that for every $\xi \in \mathbb{E}^{*}$ we have

$$
\begin{aligned}
\widehat{\nu}_{n}(\xi) & =\exp \left\{-\int_{\mathbb{E}} \int_{1 / n}^{\infty}\left(1-e^{i \xi(s x)}\right) s^{-p-1} d s \mu(d x)\right\} \\
& =\exp \left\{-\int_{\mathbb{E}}|\xi(x)|^{p} \int_{|\xi(x)| / n}^{\infty}\left(1-e^{i u \operatorname{sgn}(\xi(x))}\right) u^{-p-1} d u \mu(d x)\right\} .
\end{aligned}
$$

Let $h(u)=\left(1-e^{i u \operatorname{sgn}(\xi(x))}\right) u^{-p-1}$. Then $h$ is integrable on $[0, \infty)$ since $p \in(0,1)$, and $|h(u)|=2|\sin (u / 2)| u^{-p-1}$, thus $|h(u)| \leq u^{-p}$ for $u<1$ and $|h(u)| \leq 2 u^{-p-1}$ for $u \geq 1$. This implies that the function

$$
H_{p}(\xi(x))=\int_{0}^{\infty}\left(1-e^{i u \operatorname{sgn}(\xi(x))}\right) u^{-p-1} d u
$$

is well defined and bounded on $\mathbb{E}$, thus

$$
\widehat{\nu}_{n}(\xi) \rightarrow \exp \left\{-\int_{\mathbb{E}}|\xi(x)|^{p} H_{p}(\xi(x)) \mu(d x)\right\}=: \widehat{\gamma}_{p}(\xi) .
$$

It is easy to see that $\widehat{\gamma}_{p}$ is the characteristic function of a strictly $p$-stable random variable and the corresponding measure $\gamma_{p}$ belongs to $\mathcal{M}(\mu)$ since this class is weakly closed. Now we define

$$
\alpha_{0}=\sup \{\alpha \in(0,2]: \mathcal{M}(\mu) \text { contains a strictly } \alpha \text {-stable measure }\} .
$$

To end the proof it is enough to recall that for every $0<\beta<\alpha \leq 2$ and every strictly $\alpha$-stable measure $\gamma_{\alpha}$ the measure $\gamma_{\alpha} \circ \lambda_{\beta / \alpha}$ is strictly $\beta$-stable, where $\lambda_{\beta / \alpha}$ is the distribution of the random variable $\Theta_{\beta / \alpha}^{1 / \alpha}$, and $\Theta_{\beta / \alpha} \geq 0$ is such that $\mathbb{E} \exp \left\{-t \Theta_{\beta / \alpha}\right\}=\exp \left\{-t^{\beta / \alpha}\right\}$.

REMARK 3. Notice that if a weakly stable measure $\mu \neq \delta_{0}$ on $\mathbb{E}$ is such that $\int|\xi(x)|^{p} \mu(d x)<\infty$ for every $\xi \in \mathbb{E}^{*}$ and $p \in(0, \varepsilon)$ for some $\varepsilon \in(0,2]$ then $\mathcal{M}(\mu)$ contains a symmetric $p$-stable measure for every $p \in(0, \varepsilon)$. 
To see this it is enough to notice that if $\mu$ is symmetric, then so is the measure $\nu_{n}$ constructed in the proof of Theorem 5. Consequently,

$$
\widehat{\nu}_{n}(\xi)=\exp \left\{-\int_{\mathbb{E}}|\xi(x)|^{p} \int_{|\xi(x)| / n}^{\infty}(1-\cos u) u^{-p-1} d u \mu(d x)\right\} .
$$

Let $h(u)=(1-\cos u) u^{-p-1}$. Then $|h(u)|<u^{1-p}$ for $u<1$, and $|h(u)|<$ $2 u^{-p-1}$ for $u>1$, so $h$ is integrable on $[0, \infty)$ for every $p \in(0,2)$. For the constants

$$
H_{p}=\int_{0}^{\infty}(1-\cos u) u^{-p-1} d u
$$

we obtain

$$
\widehat{\nu}_{n}(\xi) \rightarrow \exp \left\{-H_{p} \int_{\mathbb{E}}|\xi(x)|^{p} \mu(d x)\right\},
$$

which is the characteristic function of a symmetric $p$-stable random vector.

If $\mu$ is not symmetric then we replace $\mu$ by $\mu \circ \tau$ in this construction. This is possible since $\mu \circ \tau$ is symmetric, belongs to $\mathcal{M}(\mu)$, and has the same moments as $\mu$.

REMARK 4. In the situation described in Remark 3, if $\mathbb{E}=\mathbb{R}$ then $\mathcal{M}(\mu)$ also contains a symmetric $\varepsilon$-stable random variable. Indeed, it follows from Remark 3 that

$$
\exp \left\{-H_{p} \int_{\mathbb{R}}|t x|^{p} \mu(d x)\right\}=\exp \left\{-|t|^{p} H_{p} \int_{\mathbb{R}}|x|^{p} \mu(d x)\right\}
$$

is the characteristic function of some measure from $\mathcal{M}(\mu)$. Since rescaling is admissible, $\exp \left\{-|t|^{p}\right\}$ is also the characteristic function of some measure from $\mathcal{M}(\mu)$. Now it is enough to notice that

$$
\lim _{p / \varepsilon} \exp \left\{-|t|^{p}\right\}=\exp \left\{-|t|^{\varepsilon}\right\}
$$

and use Lemma 3.

REMARK 5. There exist measures $\mu$ such that $\mu \circ \nu$ is symmetric $\alpha$-stable for some probability measure $\nu$, but $\mu$ is not weakly stable. Any measure of the form $\mu=q \delta_{-1}+(1-q) \delta_{1}$ for $q \in(0,1) \backslash\{1 / 2\}$ can serve as an example.

Lemma 10. Let $X$ be a real random variable with distribution $\mu$. If $\mu$ is weakly stable and supported on a finite set then either there exists $a \in \mathbb{R}$ such that $\mu=\delta_{a}$ or there exists $a \neq 0$ such that $\mu=\frac{1}{2} \delta_{-a}+\frac{1}{2} \delta_{a}$.

Proof. Let $X^{\prime}$ be an independent copy of $X$. Assume that $\mu \neq \delta_{a}$ for all $a \in \mathbb{R}$. Theorem 4 implies that $X$ must take on both negative and positive values with positive probability. Let $V=\{x \in \mathbb{R}: \mu(\{x\})>0\}$. By Lemma 8 we have $0 \notin V$. Let $b$ be the greatest and $-a$ the least element of $V$. Clearly, $a, b>0$. We will prove first that $a=b$. 
Assume that $b>a$. For $\lambda \in \mathbb{R}$ consider the set of values taken on by $X-\lambda X^{\prime}$ with positive probability: $V_{\lambda}=\{v-\lambda w: v, w \in V\}$. Clearly, for $\lambda \in(0,1)$ the greatest element of $V_{\lambda}$ is $b+\lambda a$, and the least is $-(a+\lambda b)$. Moreover $a+\lambda b<b+\lambda a$ (hence $b+\lambda a$ has strictly the greatest absolute value among all elements of $V_{\lambda}$ ). Since $\mu$ is weakly stable there exists a real random variable $Y_{\lambda}$ independent of $X$ and such that $Y_{\lambda} X \stackrel{d}{=} X-\lambda X^{\prime}$. One can easily see that $Y_{\lambda}$ is also finitely supported. We have $b+\lambda a \in V_{\lambda}$ so that there exist $c, d \neq 0$ such that $\mathbf{P}\left\{Y_{\lambda}=c\right\}>0, d \in V$ and $c d=b+\lambda a$. Also for any $d^{\prime} \in V$ we have $c d^{\prime} \in V_{\lambda}$, so that $\left|d^{\prime}\right|>|d|$ would imply $\left|c d^{\prime}\right|>b+\lambda a$, contrary to the fact that $b+\lambda a$ has the maximal absolute value among all elements of $V_{\lambda}$. Hence $d$ must have maximal absolute value among all elements of $V$ and therefore $d=b$ so that $c=1+\lambda a / b$.

We deduce that

$$
-\frac{a}{b}(b+\lambda a)=c \cdot(-a) \in V_{\lambda}
$$

and therefore there exist $v, w \in V$ such that $-(a / b)(b+\lambda a)=v-\lambda w$, and consequently $\lambda\left(w-a^{2} / b\right)=v+a$. Assume that $a^{2} / b \notin V$. Then the last equation may be satisfied for finitely many values of the parameter $\lambda$ only (because $v$ and $w$ can be chosen from a finite set only). It was proved for all $\lambda \in(0,1)$, however. Hence $a^{2} / b \in V$. Therefore

$$
\frac{a^{2}}{b^{2}}(b+\lambda a)=c \cdot a^{2} / b \in V_{\lambda}
$$

and again, there exist $v, w \in V$ such that $\left(a^{2} / b^{2}\right)(b+\lambda a)=v-\lambda w$ so that $\lambda\left(w+a^{3} / b^{2}\right)=v-a^{2} / b$. As before we infer that $-a^{3} / b^{2} \in V$. By iterating this reasoning we prove that $(-1)^{k+1} a^{k+1} / b^{k} \in V$ for every $k \in \mathbb{N}$. Since $0<a / b<1$ this implies that $V$ contains an infinite subset, contradicting our assumptions. The case $a>b$ is excluded in a similar way. Hence $a=b$.

Now, let $-\alpha$ be the greatest negative element and $\beta$ the least positive element of $V$. Consider $X-\lambda X^{\prime}$ for $0<\lambda<\min (\alpha, \beta) / a$. Clearly, the least positive element of $V_{\lambda}$ is $\beta-\lambda a$, whereas $-(\alpha-\lambda a)$ is the greatest negative one. Assume without loss of generality that $\beta \leq \alpha$ so that $\beta-\lambda a$ has the least absolute value among all elements of $V_{\lambda}$ (otherwise consider $-X$ instead of $X)$. Again, we choose $Y_{\lambda}$ and parameters $c, d \neq 0$ such that $\mathbf{P}\left\{Y_{\lambda}=c\right\}>0, d \in V$ and $c d=\beta-\lambda a$. We obtain $d \in\{-\alpha, \beta\}$ by a similar reasoning - no element can be both at the same side of zero as $d$ and closer to zero than $d$ because multiplying by $c$ we would get a positive element of $V_{\lambda}$ less than $\beta-\lambda a$. Hence $c \in\{(\beta-\lambda a) / \beta,-(\beta-\lambda a) / \alpha\}$. However, $c a \in V_{\lambda}$ so that there exist $v, w \in V$ such that $c a=v-\lambda w$, which means that $\lambda\left(w-a^{2} / \beta\right)=v-a$ or $\lambda\left(w+a^{2} / \alpha\right)=v+a \beta / \alpha$. Since we proved this alternative for infinitely many $\lambda$ 's and we know that $v$ and $w$ can have only 
finitely many values we infer that $a^{2} / \beta \in V$ (if $d=\beta$ ) or $-a^{2} / \alpha \in V$ (if $d=-\alpha)$.

We have proved that $V \subset[-a, a]$, so that $\beta=a$ if $d=\beta$, or $\alpha=a$ if $d=-\alpha$. Anyway, $|d|=a$ so that $|c|=\beta / a-\lambda$. Since $\{-a, a\} \subset V$ we have $\{-c a, c a\} \subset V_{\lambda}$ and therefore also $-(\beta-\lambda a) \in V_{\lambda}$. We have assumed though that $\beta-\lambda a$ has the least absolute value among all elements of $V_{\lambda}$, so in particular $-(\alpha-\lambda a) \leq-(\beta-\lambda a)$. Since $-(\alpha-\lambda a)$ is the greatest negative element of $V_{\lambda}$ we also have $-(\alpha-\lambda a) \geq-(\beta-\lambda a)$. Hence $\alpha=\beta$.

We have proved earlier that $\alpha=a$ or $\beta=a$, so finally $\alpha=\beta=a$ and the support of $\mu$ is $\{-a, a\}$. Theorem 4 implies that $\mu$ is symmetric.

Lemma 11. Let $X$ be a real random variable with distribution $\mu \neq \delta_{0}$ and let $X^{\prime}$ be its independent copy. Assume that $\mu$ is weakly stable, so that for any $\lambda \in \mathbb{R}$ there exists a real random variable $Y_{\lambda}$ independent of $X$ such that $X-\lambda X^{\prime} \stackrel{d}{=} Y_{\lambda} X$. If $X$ is symmetric, assume additionally that $Y_{\lambda} \geq 0$ a.s. Then the map

$$
\lambda \mapsto \mathcal{L}\left(Y_{\lambda}\right)
$$

is well defined and continuous on $\mathbb{R}$.

Proof. The existence and uniqueness of distribution of $Y_{\lambda}$ follows from Theorems 2 and 3 . We only need to prove that $\lambda_{n} \rightarrow \lambda$ implies that $Y_{\lambda_{n}} \stackrel{d}{\rightarrow} Y_{\lambda}$ as $n \rightarrow \infty$. Suppose not. Then we can find $\varepsilon>0$ and a subsequence $\left\{n_{k}\right\}$ such that for any $k$ the law of $Y_{\lambda_{n_{k}}}$ is $\varepsilon$-separated from the law of $Y_{\lambda}$ in Lévy's metric. Since

$$
Y_{\lambda_{n_{k}}} X \stackrel{d}{=} X-\lambda_{n_{k}} X^{\prime} \stackrel{d}{\rightarrow} X-\lambda X^{\prime} \stackrel{d}{=} Y_{\lambda} X
$$

by Lemma 2 we can choose a subsequence $\left\{n_{k_{l}}\right\} \subset\left\{n_{k}\right\}$ such that $Y_{\lambda_{n_{k_{l}}}} \stackrel{d}{\rightarrow} Z$ for some real random variable $Z$ as $l \rightarrow \infty$. Hence $\mathcal{L}(Z) \neq \mathcal{L}\left(Y_{\lambda}\right)$. Moreover $Z \geq 0$ a.s. if $X$ is symmetric because then all $Y_{\lambda_{n}}$ 's are nonnegative a.s.

On the other hand, $Z^{\prime} X \stackrel{d}{=} Y_{\lambda} X$, where $Z^{\prime}$ is a copy of $Z$ independent of $X$ since the map $\lambda \mapsto \mathcal{L}\left(X-\lambda X^{\prime}\right)$ is continuous. Therefore $Y_{\lambda} \stackrel{d}{=} Z^{\prime} \stackrel{d}{=} Z$, by Theorems 2 and 3 (or by Remark 1). The contradiction obtained ends the proof.

Remark 6. Let $\alpha \in[1,2]$. Note that if $X$ is a random variable with a weakly stable distribution $\mu$ and $\mathbf{E}|X|^{p}<\infty$ for all $p \in(0, \alpha)$ then

$$
1+|\lambda| \geq \begin{cases}\left|Y_{\lambda}\right| \text { a.s. } & \text { if } \alpha=2 \\ \left\|Y_{\lambda}\right\|_{\alpha} & \text { if } \alpha<2 .\end{cases}
$$

Indeed, by Theorem 5 there exists $\Theta$ independent of $X$ such that $X \Theta$ is strictly $\alpha$-stable. If $\alpha<2$ then $\mathbf{E}|X \Theta|^{\beta}<\infty$ for every $\beta<\alpha$, thus $\mathbf{E}|X|^{\beta}<\infty$ for every $\beta<\alpha$. If $\alpha=2$ then $X \Theta$ is Gaussian so $\mathbf{E}|X|^{\beta}<\infty$ 
for every $\beta>0$. Now it is enough to notice that for $\beta \geq 1$ we have $\left\|Y_{\lambda}\right\|_{\beta}\|X\|_{\beta}=\left\|Y_{\lambda} X\right\|_{\beta}=\left\|X-\lambda X^{\prime}\right\|_{\beta} \leq\|X\|_{\beta}+|\lambda|\left\|X^{\prime}\right\|_{\beta}=(1+|\lambda|)\|X\|_{\beta}$.

The case $\beta=\alpha$ can be obtained by observing that $\left\|Y_{\lambda}\right\|_{\alpha}=\lim _{\beta \rightarrow \alpha^{-}}\left\|Y_{\lambda}\right\|_{\beta}$. If $\alpha=2$ the inequality holds for all $\beta \geq 1$, which implies that $\left\|Y_{\lambda}\right\|_{\infty} \leq$ $1+|\lambda|$.

Lemma 12. Let $X$ be a real random variable with distribution $\mu$. If $\mu$ is weakly stable and supported on a countable set then there exists $a \in \mathbb{R}$ such that $\mu=\delta_{a}$ or there exists $a \neq 0$ such that $\mu=\frac{1}{2} \delta_{-a}+\frac{1}{2} \delta_{a}$.

Proof. Assume that the support of $\mu$ is an infinite countable set. For $\lambda \in(0,1)$ we have $X-\lambda X^{\prime} \stackrel{d}{=} Y_{\lambda} X$, where $X^{\prime}$ and $Y_{\lambda}$ are defined as in the preceding lemma (so that if $X$ is symmetric then $Y_{\lambda} \geq 0$ a.s.). By Lemma 11, $Y_{\lambda} \stackrel{d}{\rightarrow} Y_{0}=1$ as $\lambda \rightarrow 0$. Let

$$
\mu=\sum_{n=1}^{\infty} p_{n} \delta_{x_{n}},
$$

where $x_{n}$ 's are nonzero (by Lemma 8) and pairwise different, and $\left(p_{n}\right)_{n=1}^{\infty}$ is a nonincreasing sequence of positive numbers. Let

$$
M=\left\{\frac{x_{i}-x_{j}}{x_{k}-x_{l}}: k \neq l\right\} .
$$

Clearly, $M$ is a countable set. We see that for $\lambda \notin M$ the equality $x_{k}-\lambda x_{i}$ $=x_{l}-\lambda x_{j}$ implies $i=j$ and $k=l$. Finally, let $N \in \mathbb{N}$ be such that $\sum_{n>N} p_{n} \leq p_{1}^{2} / 2$. Then for $\lambda \in(0,1) \backslash M$ we have

$$
\begin{aligned}
p_{1}^{2} & =\mathbf{P}\left\{X=x_{1}, X^{\prime}=x_{1}\right\}=\mathbf{P}\left\{X-\lambda X^{\prime}=x_{1}-\lambda x_{1}\right\} \\
& =\mathbf{P}\left\{Y_{\lambda} X=x_{1}(1-\lambda)\right\}=\sum_{n=1}^{\infty} \mathbf{P}\left\{Y_{\lambda}=\frac{x_{1}}{x_{n}}(1-\lambda)\right\} \cdot p_{n} \\
& \leq \mathbf{P}\left\{Y_{\lambda}=1-\lambda\right\} \cdot p_{1}+\frac{p_{1}^{2}}{2}+\sum_{n=2}^{N} \mathbf{P}\left\{\frac{Y_{\lambda}}{1-\lambda}=\frac{x_{1}}{x_{n}}\right\} \cdot p_{n},
\end{aligned}
$$

and the summands for $2 \leq n \leq N$ tend to zero as $\lambda \rightarrow 0$ (since $\frac{Y_{\lambda}}{1-\lambda} \stackrel{d}{\rightarrow} 1$ ) so that

$$
\liminf _{\lambda \rightarrow 0, \lambda \in(0,1) \backslash M} \mathbf{P}\left\{Y_{\lambda}=1-\lambda\right\} \geq p_{1} / 2 .
$$

On the other hand, for $\lambda \in(0,1) \backslash M$ and $k \in \mathbb{N}$ we have

$$
\begin{aligned}
p_{k}^{2} & =\mathbf{P}\left\{X=x_{k}, X^{\prime}=x_{k}\right\}=\mathbf{P}\left\{X-\lambda X^{\prime}=x_{k}(1-\lambda)\right\} \\
& =\mathbf{P}\left\{Y_{\lambda} X=x_{k}(1-\lambda)\right\} \geq \mathbf{P}\left\{Y_{\lambda}=1-\lambda\right\} \cdot p_{k},
\end{aligned}
$$


so that

$$
\limsup _{\lambda \rightarrow 0, \lambda \in(0,1) \backslash M} \mathbf{P}\left\{y_{\lambda}=1-\lambda\right\} \leq p_{k} .
$$

Hence $p_{k} \geq p_{1} / 2$ for any $k \in \mathbb{N}$ and $\sum_{k=1}^{\infty} p_{k}=\infty$, which is clearly not possible. This proves that $\mu$ has finite support and the assertion follows from Lemma 10.

THEOREM 6. Let $\mu$ be a weakly stable probability measure on a separable Banach space $\mathbb{E}$. Then either there exists $a \in \mathbb{E}$ such that $\mu=\delta_{a}$, or there exists $a \in \mathbb{E} \backslash\{0\}$ such that $\mu=\frac{1}{2} \delta_{-a}+\frac{1}{2} \delta_{a}$, or $\mu(\{a\})=0$ for all $a \in \mathbb{E}$.

Proof. Assume first that $\mathbb{E}=\mathbb{R}$. One can express $\mu$ as $p \mu_{1}+(1-p) \mu_{2}$, where $p \in[0,1], \mu_{1}$ is a discrete probability measure and $\mu_{2}(\{x\})=0$ for any $x \in \mathbb{R}$. The case $p=0$ is trivial, so assume that $p>0$. Lemma 9 implies that $\mu_{1}$ is weakly stable, and therefore by Lemma $12, \mu_{1}=\delta_{a}$ for some $a \in \mathbb{R}$ or $\mu_{1}=\frac{1}{2} \delta_{-a}+\frac{1}{2} \delta_{a}$ for some $a \neq 0$.

CASE 1: $\mu_{1}=\delta_{a}$. If $a=0$ then by Lemma 8 we have $p=1$ and the proof is finished. If $a \neq 0$, note that for $\lambda \in(0,1)$ the random variable $X-\lambda X^{\prime} \stackrel{d}{=} Y_{\lambda} X$ has exactly one atom with mass $p^{2}$ at $(1-\lambda) a$. Hence $Y_{\lambda}$ has an atom with mass $p$ at $1-\lambda$. Since $Y_{\lambda} \stackrel{d}{\rightarrow} Y_{1}$ as $\lambda \rightarrow 1$ we have $\mathbf{P}\left\{Y_{1}=0\right\} \geq p$, and therefore $\mathbf{P}\left\{X-X^{\prime}=0\right\}=\mathbf{P}\left\{Y_{1} X=0\right\} \geq p$. On the other hand, $\mathbf{P}\left\{X-X^{\prime}=0\right\}=\mathbf{P}\left\{X=X^{\prime}\right\}=p^{2}$ because $X$ has only one atom, at $a$. Hence $p^{2} \geq p$ so that $p=1$.

CASE 2: $\mu_{1}=\frac{1}{2} \delta_{-a}+\frac{1}{2} \delta_{a}$ for some $a \neq 0$. For $\lambda \in(0,1)$ the random variable $X-\lambda X^{\prime} \stackrel{d}{=} Y_{\lambda} X$ has exactly four atoms, with mass $p^{2} / 4$ each, at $\pm(1-\lambda) a$ and $\pm(1+\lambda) a$. Hence $Y_{\lambda}$ has atoms with total mass $p / 2$ at $\pm(1-\lambda)$ (and atoms with total mass $p / 2$ at $\pm(1+\lambda)$ ). Since $Y_{\lambda} \stackrel{d}{\rightarrow} Y_{1}$ as $\lambda \rightarrow 1$ we have $\mathbf{P}\left\{Y_{1}=0\right\} \geq p / 2$, and therefore $\mathbf{P}\left\{X-X^{\prime}=0\right\}=\mathbf{P}\left\{Y_{1} X=0\right\} \geq p / 2$. On the other hand, $\mathbf{P}\left\{X-X^{\prime}=0\right\}=\mathbf{P}\left\{X=X^{\prime}=a\right\}+\mathbf{P}\left\{X=X^{\prime}=-a\right\}$ $=p^{2} / 2$ so that $p^{2} / 2 \geq p / 2$ and $p=1$.

Let now $\mathbb{E}$ be an arbitrary separable Banach space. By making use of the above result for real random variables $\xi(X)$, where $\xi \in \mathbb{E}^{*}$, we can easily finish the proof.

REMARK 7. Let $X$ be a given random variable. It may happen that for some $a, b \in \mathbb{R}$ there exists a random variable $Q_{a, b}$ independent of $X$ such that $a X+b X^{\prime} \stackrel{d}{=} X Q_{a, b}$, and for some other $a, b$ such a random variable does not exist.

Consider, for example, $X$ with exponential distribution and characteristic function $(1-i t)^{-1}$. It follows from Theorem 4 that the class $\mathcal{M}\left((1-i t)^{-1}\right)$ is not closed under convolutions. However, if $a, b \in \mathbb{R}$ are such that $a b<0$ 
then the characteristic function of $a X+b X^{\prime}$ can be written as

$$
\mathbf{E} \exp \left\{i\left(a X+b X^{\prime}\right) t\right\}=\frac{1}{1-i a t} \frac{1}{1-i b t}=\int \frac{1}{1-i s t} \lambda(d s),
$$

where $\lambda(\{a\})=p=1-\lambda(\{b\})$, with $p=a /(a-b)$. This means that $a X+b X^{\prime} \stackrel{d}{=} X Q_{a, b}$, where $Q_{a, b}$ is independent of $X$ and has distribution $\lambda$.

Assume that for some $a, b>0$ there exists $Q \geq 0$ such that $a X+b X^{\prime} \stackrel{d}{=}$ $X Q$. Then the density $g$ of $a X+b X^{\prime}$ can be written as

$$
g(x)=\int_{0}^{\infty} e^{-x / s} s^{-1} \mathcal{L}(Q)(d s) .
$$

On the other hand, we have

$$
g(x)=\int_{0}^{\infty} e^{-x / s} s^{-1} \lambda(d s)
$$

The uniqueness of the Laplace transform for signed $\sigma$-finite measures implies that $\mathcal{L}(Q)=\lambda$, which is impossible since $\mathcal{L}(Q)$ is a probability measure while $\lambda$ is a signed measure only. Similar arguments can be used for $a, b<0$. Finally, if $a b>0$ then $a X+b X^{\prime}$ cannot have the same distribution as $X Q$ for any random variable $Q$ independent of $X$.

\section{References}

[1] S. Cambanis, R. Keener and G. Simons, On $\alpha$-symmetric distributions, J. Multivariate Anal. 13 (1983), 213-233.

[2] J. Kucharczak and K. Urbanik, Quasi-stable functions, Bull. Acad. Polon. Sci. Sér. Sci. Math. Astronom. Phys. 22 (1974), 263-268.

[3] - - - Transformations preserving weak stability, Bull. Polish Acad. Sci. Math. 34 (1986), 475-486.

[4] L. H. Loomis, An Introduction to Abstract Harmonic Analysis, Van Nostrand, Toronto, 1953.

[5] G. Mazurkiewicz, When a scale mixture is equivalent with rescaling, J. Math. Sci. (New York) 111 (2002), 3851-3853.

[6] J. K. Misiewicz, Positive definite functions on $l^{\infty}$, Statist. Probab. Lett. 8 (1989), $255-260$.

[7] - Elliptically contoured measures on $R^{\infty}$, Bull. Polish Acad. Sci. Math. 30 (1982), 283-290.

[8] G. Samorodnitsky and M. S. Taqqu, Stable non-Gaussian Random Processes. Stochastic Models with Infinite Variance, Chapman \& Hall, 1994.

[9] I. J. Schoenberg, Metric spaces and completely monotonic functions, Ann. of Math. 38 (1938), 811-841.

[10] K. Urbanik, Remarks on $\mathcal{B}$-stable probability distributions, Bull. Polish Acad. Sci. Math. 24 (1976), 783-787.

[11] V. Vol'kovich, On symmetric stochastic convolutions, J. Theoret. Probab. 5 (1992), 417-430. 
[12] V. Vol'kovich, Infinitely divisible distributions in algebras with stochastic convolution, J. Soviet Math. 40 (1988), 459-467.

[13] - Multidimensional $\mathcal{B}$-stable distributions and realizations of generalized convolutions, in: Stability Problems of Stochastic Models (Moscow, 1984), VNIISI, Moscow, 1984, 40-54 (in Russian).

Department of Mathematics, Informatics and Econometry University of Zielona Góra

Podgórna 50

65-246 Zielona Góra, Poland

E-mail: j.misiewicz@wmie.uz.zgora.pl

Institute of Mathematics

Warsaw University

Banacha 2

02-097 Warszawa, Poland

E-mail: koles@mimuw.edu.pl

Institute of Mathematics

University of Wrocław

Pl. Grunwaldzki 2/4

50-384 Wrocław, Poland

E-mail: urbanik@math.uni.wroc.pl

Received December 2, 2002

Revised version December 7, 2004

(5089) 